\title{
Variabilidade Temporal do Sistema da Corrente do Brasil e da Corrente de Contorno Intermediário em $23^{\circ} \mathrm{S}$
}

\author{
Matheus Laviola da Silva ${ }^{*}$, Leonardo Carvalho de Jesus ${ }^{1}$, Fernando Camilo ${ }^{1}$ y Julio Aquije ${ }^{* *} 1$ \\ ${ }^{1}$ Universidade Federal do Espírito Santo, Vitória, Brazil
}

Recibido 12 febrero 2020 - Aceptado 07 diciembre 2020

\begin{abstract}
Resumo
O sistema da Corrente do Brasil (CB) é caracterizado pelo meandramento e a geração de vórtices associados à corrente. E, constitui o principal sistema de correntes ao largo da região sudeste brasileira, região de enorme importância econômica, biológica e geopolítica para o Brasil. A dinâmica da corrente do Brasil é influenciada principalmente pelo local de formação, a batimetria, e a instabilidade baroclínica e barotrópica. O cálculo do transporte volumétrico da Corrente do Brasil (CB) e da Corrente de Contorno Intermediária (CCI) em $23^{\circ} \mathrm{S}$ é realizado neste trabalho usando dados de reanálises do modelo HYCOM-NCODA. A série histórica de vazão foi analisada usando a estatística básica e a transformada de wavelet. Os resultados do espectro de wavelet mostram um ciclo anual bem marcado na $\mathrm{CB}$ e na CCI. Assim como padrões de variação diferentes entre a CB e a CCI, sendo encontrado nesta última maior variabilidade no espectro.
\end{abstract}

Palavras Chave: CB, CCI, transporte de volume, variabilidade.

\section{Temporal variability of the Brazil and the Intermediate Contour current's system at $23^{\circ} \mathrm{S}$}

\begin{abstract}
The Current of Brazil (CB) system is associated with the movement and generation of eddies associated with the current. And, to establish the main chain system off the Brazilian region, a region of enormous economic, biological and geopolitical importance for Brazil. The amount of current in Brazil is mainly influenced by local training, bathymetry and baroclinic and barotropic instability. The calculation of the volumetric transport of the Brazil Current (CB) and the Intermediate Contour Current (CCI) at $23^{\circ} \mathrm{S}$ is performed in this work using data from reanalysis of the HYCOM-NCODA model. A historical leak series was analyzed using basic statistics and a wavelet transformation. The results of the wavelet spectrum show a well-marked annual cycle in CB and CCI. As well as the different variation patterns between $\mathrm{CB}$ and $\mathrm{CCI}$, the latter found greater variability in the spectrum.
\end{abstract}

Keywords: RIF Journal, publication rules.

\section{Introdução}

No Atlântico Sul, a Corrente do Brasil (CB) é uma corrente de contorno oeste forçada na camada superficial pela alta subtropical (ASAS) e caracterizada por ter uma temperatura e salinidade em torno dos $26^{\circ} \mathrm{C}$ e 36 ups, respectivamente (Lorenzzetti et al., 2006). A CB se forma próxima à latitude $16^{\circ} \mathrm{S}$ (Stramma \& England, 1999), após a bifurcação do ramo sul da Corrente Sul Equatorial (CSEs).

\footnotetext{
*matheus.bonjour@gmail.com

**julio.chacaltana@ufes.br
}

À medida que a CB escoa para sul, as massas da Água Central do Atlântico Sul (ACAS) e da Água Intermediária Antártica (AIA) são incorporadas em seu transporte, causando o aprofundamento da CB. A ACAS se incorpora à $\mathrm{CB}$ em torno dos $20^{\circ} \mathrm{S}$. Nessa latitude, se encontram os montes e bancos submarinos da cadeia Vitoria-Trindade, responsáveis pela bifurcação da ACAS na região da picnoclina. (Stramma \& England, 1999). Após essa bifurcação, parte da ACAS escoa para sul, incorporando-se à $\mathrm{CB}$ e parte para norte, incorporada 
pela Corrente de Contorno Intermediária (CCI).

Em torno dos $28^{\circ} \mathrm{S}$, a AIA se bifurca (Boebel et al., 1999) na região da subpicnoclina, entre as profundidades de $500 \mathrm{~m}$ e $1000 \mathrm{~m}$. Após essa bifurcação, a porção que escoa para norte dá origem à CCI e a porção que escoa para sul é incorporada pela CB.

Duas características são responsáveis por tornar o sistema da CB um sistema de correntes único no planeta: o aprofundamento da CB em direção ao sul (como mostrado anteriormente), e o elevado cisalhamento vertical decorrente da interação entre a CB e a CCI que ocorre aproximadamente entre $15^{\circ} \mathrm{S}$ e $25^{\circ} \mathrm{S}$.

A segunda característica é a responsável pelo elevado caráter baroclínico do sistema da CB, como encontrado por Silveira et al. (2004, 2008) e Rocha et al. (2014). Este último associado às características morfológicas (como a mudança de orientação da costa ao sul de Cabo Frio-RJ), são responsáveis pela elevada ocorrência de meandros e vórtices na região. A elevada ocorrência de processos internos, como meandros e vórtices, juntamente com fenômenos remotos, como o ENSO (El Niño-Southern Oscillation) e a convergências das Malvinas e Antártica, são os forçantes que devem ser considerados no estudo da variabilidade no sistema da $\mathrm{CB}$.

\section{Materiais e Métodos}



Figura 1: Transecto na região de $23^{\circ} \mathrm{S}$ adjacente à Cabo Frio-RJ.

Para o estudo da variabilidade do sistema formado pela $\mathrm{CB}$ e CCI foram utilizados dados de reanálises do modelo HYCOM + NCODA de $1 / 12^{\circ}$ de resolução espacial horizontal e 40 níveis na vertical. Dados diários de 5 variáveis, dentre as quais as componentes meridional e zonal de velocidade, a salinidade, a temperatura e a elevação da superfície do mar, são disponibilizados para diferentes profundidades. A vazão ou transporte de volume da CB e CCI foi calculado para um período 20 anos compreendido entre o 01 de janeiro de 1993 a 31 de dezembro de 2012. Os dados diários de velocidade zonal e meridional foram interpolados à posição do transecto de $135 \mathrm{~km}$ de comprimento, perpendicular à linha de costa e localizado em $23^{\circ} \mathrm{S}$ na região de Cabo Frio, como é mostrado na Figura 1. O cálculo diário do transporte de volume $(\mathrm{Tv})$ foi realizado através da equação integral da vazão dada pela Equação (1), em que $(\vec{n})$ é o vetor normal unitário ao transecto e $(\vec{u})$ é o vetor velocidade. $\mathrm{Na}$ integração, a CB foi considerada desde a superfície d'água até a profundidade de $500 \mathrm{~m}$ e para a CCI foi considerada a profundidade de $500 \mathrm{~m}$ até $1200 \mathrm{~m}$. Em ambos os casos, o vetor normal unitário aponta para o norte.

$$
T v=\int_{S}(\vec{u} . \hat{n}) d S
$$

A série diária do transporte de volume da CB foi analisada por meio de parâmetros estatísticos básicos, calculando a média histórica e seu desvio padrão. Seguidamente, foi calculada a média mensal do transporte de volume e sua respectiva anomalia, esta última foi calculada tendo como referência a média histórica de cada mês. Finalmente, o espectro de wavelet foi calculado usando como função mãe a wavelet de Morlet, espectro que permite avaliar as frequências de ocorrências e suas energias na série temporal de transporte volumétrico da CB. A partir desse estudo, foram analisadas as variações do transporte da CB no período de 20 anos. Um procedimento semelhante é realizado para a série diária do transporte de volume da CCI.

\section{Resultados e Discussões}

A série histórica da média mensal do transporte volumétrico da CB é mostrada na Figura 2-a e da CCI mostrada na Figura 2-b. A média de cada mês e seus respectivos desvios padrões do transporte volumétrico da $\mathrm{CB}$ e da CCI são mostradas respectivamente na Figura 3-a e Figura 3-b. Nas Figuras 4-a e Figura 4-b, são mostrados respectivamente o espectro de ondaleta (Transformada de ondaleta de Morlet) e o espectro global de ondaleta da $\mathrm{CB}$, enquanto que os espectros referentes à CCI são mostrados nas figuras 4-c e Figura 4-d. A linha contínua em preto nas Figuras 4-a e Figura 4-c delimita o cone de influência.

O transporte de volume da CB mostrado na Figura 2-a está de acordo com o encontrado em literatura, por exemplo em Silveira et al. (2004). Como se pode observar na série temporal da CB, há uma clara tendência de diminuição do transporte volumétrico com o passar dos anos. Já o transporte de volume da CCI, mostrado na Figura 2-b parece ser menor que o valor da média esperada para a região ( $4 \mathrm{~Sv}$ ). Ao longo de toda série temporal, os dados do HYCOM-NCODA mostram que a bifurcação 
da AIA ocila entre $25^{\circ} \mathrm{S}$ e $29^{\circ} \mathrm{S}$, com média tempotal entre $26.5^{\circ} \mathrm{S}$ e $27.5^{\circ} \mathrm{S}$, o que pode gerar menores valores de transporte volumétrico para a região analisada.

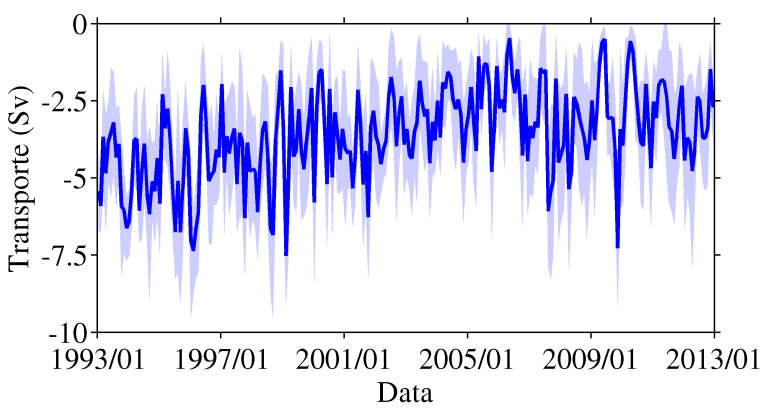

(b)



Figura 2: Medias mensais e seus respectivos desvios padrão do transporte da CB (a) e transporte da CCI (b).

a)

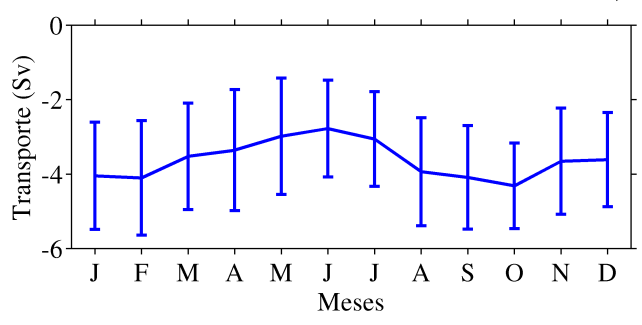

b)

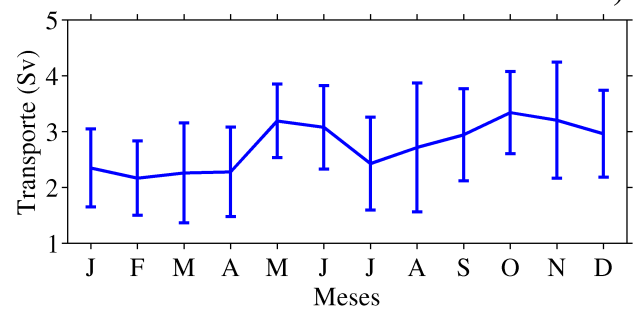

Figura 3: Médias históricas de cada mês do transporte da CB (a) e da CCI (b), com seus respectivos desvios padrão. a)

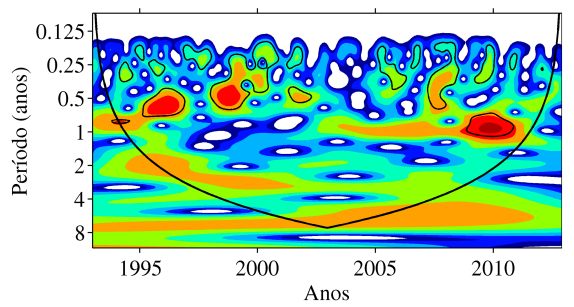

c)

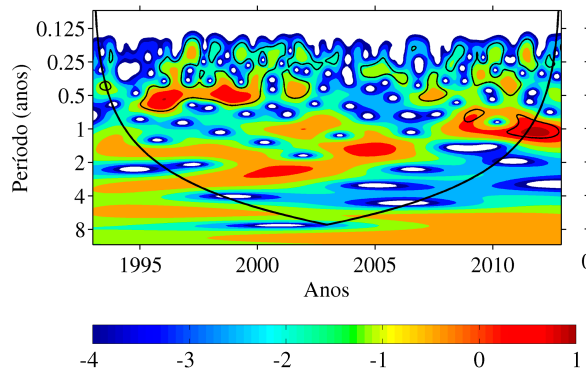

b)

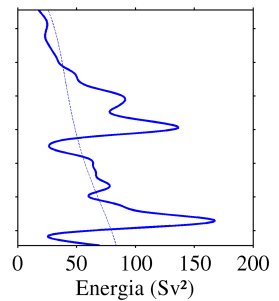

d)

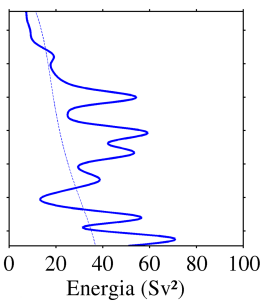

Figura 4: Espectro de ondaleta Morlet do transporte da CB (a) e da CCI (c); Espectro global de ondaleta da CB (b) e da CCI (d).

Uma análise da média histórica mensal do transporte de volume da CB e da CCI, mostrada na Figura 3, revelam oscilações de um ciclo anual bem definido para a CB, e mais de um cliclo anual para a CCI. Na Figura 3-a, pode ser observado que no mês de junho ocorre o menor transporte de volume na $\mathrm{CB}$, enquanto que no mês de outubro ocorre o maior transporte de volume. Para a CCI, pode ser observado na Figura 3-b que os meses com maior transporte de volume são maio e outubro, enquanto seu enfraquecimento é notado nos meses de fevereiro e julho.

A análise do espectro de energia global da CB (Figura 4-b) coloca em evidência dois picos de energia. $\mathrm{O}$ de maior energia tem período bem próximo de um ano seguido por um pico de menor energia com período de meio ano. A Figura 4-a mostra que pico energético com período de meio ano está bem representado pelos eventos que aconteceram nos anos de 1996 e 1999. Próximo ao período de um ano, se pode visualizar eventos que iniciaram no ano de 2004 e terminaram em 2011, com sua maior energia bem definida no ano de 2010. O espectro de energia global da CCI apresenta três picos energéticos bem definidos, como observado na Figura 4-d. O picos de maior energia estão associados a um período de um ano seguido por 2 picos de igual energia. Estes picos ocorrem em período bem definido de meio ano e próximo de dois anos. Na 4-c, é possível observar que os eventos de maior energia que ocorreram no intervalo de 1995 a 2000 estão associados ao pico de período de meio ano. Já para o pico de período de um ano, os eventos energéticos estão associados à faixa de tempo que ocorreram nos anos 
de 2001 a 2003 e, entre os anos de 2008 e 2011, sendo o ano de 2011 o de maior energia. Finalmente, para o pico de período aproximado a dois anos, os eventos de maior energia que contribuem estão associados à faixa de tempo entre os anos de 2000 e 2001 e, entre os anos de 2003 e 2005

\section{Conclusões}

Os dados do modelo HYCOM-NCODA conseguem representar o transporte volumétrico da $\mathrm{CB}$ em concor- dância com o relatado pela literatura, o que não tem sido encontrado para o transporte de volume da CCI que foi menor do que o esperado. Um dos motivos para essa diferença no transporte de volume da CCI pode ser a oscilação espacial da localização da bifurcação da AIA. Como também, a alta dinâmica do sistema de corrente que com o enfraquecimento da CB ocasiona uma ascensão vertical do núcleo da CCI. As médias históricas dos meses, mostraram ciclos anuais bem definidos da $\mathrm{CB}$ e CCI. Porém, a análise de frequência dos espectros globais de ondaleta mostram outras componentes de meio e dois ciclos por ano.

\section{Referencias}

[Boe99] Boebel, O., Davis, R., Ollitraut, M., Peterson, R., Richard, P., Schmid, C., Zenk, W. (1999). The intermediate depth circulation of the Western South Atlantic. Geophysical Research Letters. 26(21), 3329-3332.

[Lor06] Lorenzzetti, J., Kampel, M., Bentz, C., Torres Jr., A. (2006). A meso-scale Brazil current frontal eddy: observations by asar, radarsat- 1 complemented with visible and infrared sensors, in situ data, and numerical modeling. Proceedings of SEASAR, 23-26

[Roc14] Rocha, C., Silveira, I., Castro, B., Lima, J. (2014). Vertical structure, energetics, and dynamics of the Brazil Current System at $22^{\circ} \mathrm{S}-28^{\circ} \mathrm{S}$. Journal of Geophysical Research, 119, 52-69.
[Sil04] Silveira, I., Calado, L., Castro, B., Cirano, M., Lima, J., Mascarenhas, A. (2004). On the baroclinic structure of the Brazil Current Intermediate Western Boundary Current System. Geophysical Research Letters. 31(14), L14308

[Sil08] Silveira, I., Lima, J., Schmidt, A., Ceccopieri, W., Sartori, A., Franscisco, C., Fontes, R. (2008). Is the meander growth in the Brazil Current system of Southeast Brazil due to baroclinic instability?. Dynamics of Atmosphere and Oceans 45, 187207

[Str99] Stramma, L. and England, M. (1999). On the water masses and mean circulation of the South Atlantic Ocean. Journal of Geophysical Research, 104 (C9), 20.863-20.883. 